\title{
Modelling the HIV-Associated TB Epidemic and the Impact of Interventions Aimed at Epidemic Control
}

\author{
P. J. Dodd, C. Pretorius, and B. G. Williams
}

\begin{abstract}
In this chapter, we focus on mathematical models of tuberculosis epidemiology (TB) that include interactions with HIV and an explicit representation of transmission. We review the natural history of TB and illustrate how its features are simplified and incorporated in mathematical models. We then review the ways HIV influences the natural history of TB, the interventions that have been considered in models, and the way these individual-level effects are represented in models. We then go on to consider population-level effects, reviewing the TB/HIV modelling literature. We first review studies whose focus was on purely epidemiological modelling, and then studies whose focus was on modelling the impact of interventions. We conclude with a summary of the uses and achievements of TB/ HIV modelling and some suggested future directions.
\end{abstract}

Keywords Tuberculosis · Human immunodeficiency virus · Mathematical modelling · Transmission modelling · TB/HIV · Epidemiological modelling · Economic evaluation $\cdot$ Natural history $\cdot$ Epidemiology

The original version of this chapter was revised. The correction to this chapter is available at https://doi.org/10.1007/978-3-030-29108-2_14

\section{P. J. Dodd $(\bowtie)$}

School of Health and Related Research, University of Sheffield, Sheffield, UK

e-mail: p.j.dodd@ sheffield.ac.uk

C. Pretorius

Avenir Health, Glastonbury, CT, USA

B. G. Williams

South African Centre for Epidemiological Modelling and Analysis,

University of Stellenbosch, Stellenbosch, South Africa 


\section{Introduction}

The emergence of the HIV pandemic in sub-Saharan Africa and the simultaneous rise in the incidence of tuberculosis (TB) in this region is a stark reminder of the importance of HIV-epidemiology for modelling TB (Fig. 1). As HIV prevalence increased during the 1990s, the per-capita TB notification rate increased severalfold in some countries. As the HIV epidemics matured and the mean duration of HIV-infection grew longer, the mean level of population immunocompetence declined, and the strength of association increased. By the late 1990s for example, over $70 \%$ of TB notifications in Zimbabwe were in people living with HIV (PLHIV) [1]. Comparing this proportion to the HIV infection prevalence in the population without TB yields an odds ratio of around 10, which can also be interpreted as an average incidence rate ratio for developing TB if infected with HIV [2].

With the wider roll-out of antiretroviral therapy for HIV (ART) beginning around 2004, initially provided to those meeting certain CD4 count thresholds and now recommended for all PLHIV, these population-level associations between HIV and TB incidence began to weaken. As the epidemic declined in some countries, including Zimbabwe and Ethiopia, and ART was rolled out very aggressively, as in Botswana and South Africa, the TB notification rates declined but not to the preHIV levels. Today, around $10 \%$ of TB is thought to be HIV-associated globally (74\% of this in sub-Saharan Africa) [3]. A higher, but more uncertain, proportion of TB deaths are HIV-associated, but vital registration cause-of-death coding rules and frequent comorbidities make such estimates more difficult. However, the fairly constant HIV prevalence and still-elevated risks of developing TB even among PLHIV on ART means that HIV will continue to affect TB epidemiology for decades to come and remain a crucial aspect of modelling in high-HIV burden settings.
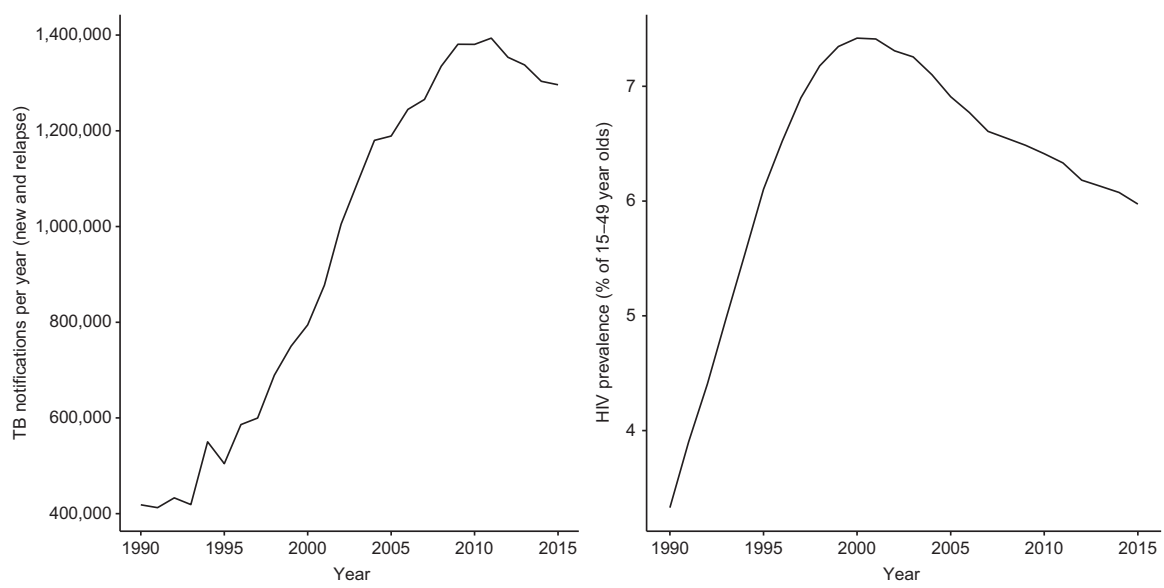

Fig. 1 TB and HIV in sub-Saharan Africa between 1990 and 2015: total tuberculosis notifications reported to the World Health Organisation (WHO) by countries in the WHO African region (left); UNAIDS estimates of HIV prevalence in 15-49 year olds (right) 


\section{Focus of This Chapter}

The use of mathematical modelling as a tool in public health for understanding the behaviour of complex systems, and of assessing the impact and cost-effectiveness of interventions has grown. For an infectious disease like TB, making quantitative predictions about the population-level impact of interventions requires models that explicitly account for the transmission process. Without including transmission, there is no way of assessing the indirect benefits interventions may have through onward cases averted. For interventions whose benefit accrues mainly or wholly at the individual-patient level, the standard static modelling approaches of health economics are likely to be appropriate, but for interventions that are likely to reduce transmission, static models may substantially underestimate benefits from indirect effects. In this chapter, we focus explicitly on dynamic models, that is populationlevel transmission models where the risks of infection depend on the prevalence of infectious cases and may change in response to interventions.

We first review the natural history and transmission epidemiology of TB, and describe in nontechnical terms the way these features are approximated and abstractly represented in transmission models. We describe the way HIV affects individuals' natural history of TB and the approaches that have been used to model these effects. We highlight a number of interventions and describe how these have been allowed for in TB models, and we review the literature on modelling the impact of these interventions on TB at a population-level. We conclude by critically reflecting on the achievements and limitations of the work to date and suggest future directions.

\section{TB Modelling Without HIV}

Before going on to consider how TB/HIV is modelled, it is important to understand how TB is usually represented in transmission models and what modelling techniques are used. In this section, we introduce compartmental models of TB and step through various aspects of TB natural history, discussing how modellers approach incorporating these into mathematical models.

\section{Modelling Approaches}

We will centre our description of TB modelling on Fig. 2, which is largely based on the early work of Dye and colleagues [4] (see also the book by Dye [5]), which, with variations largely reflecting different model purpose, has become the canonical basis for TB transmission models. Different disciplines have brought different techniques, focus and approaches to the analysis, with TB/HIV modelling papers appearing in journals ranging from mathematical biology focussed on formally characterizing properties of specific models with abstract applications, through population biology/ecology studies aimed at exploring actual or potential behaviour 


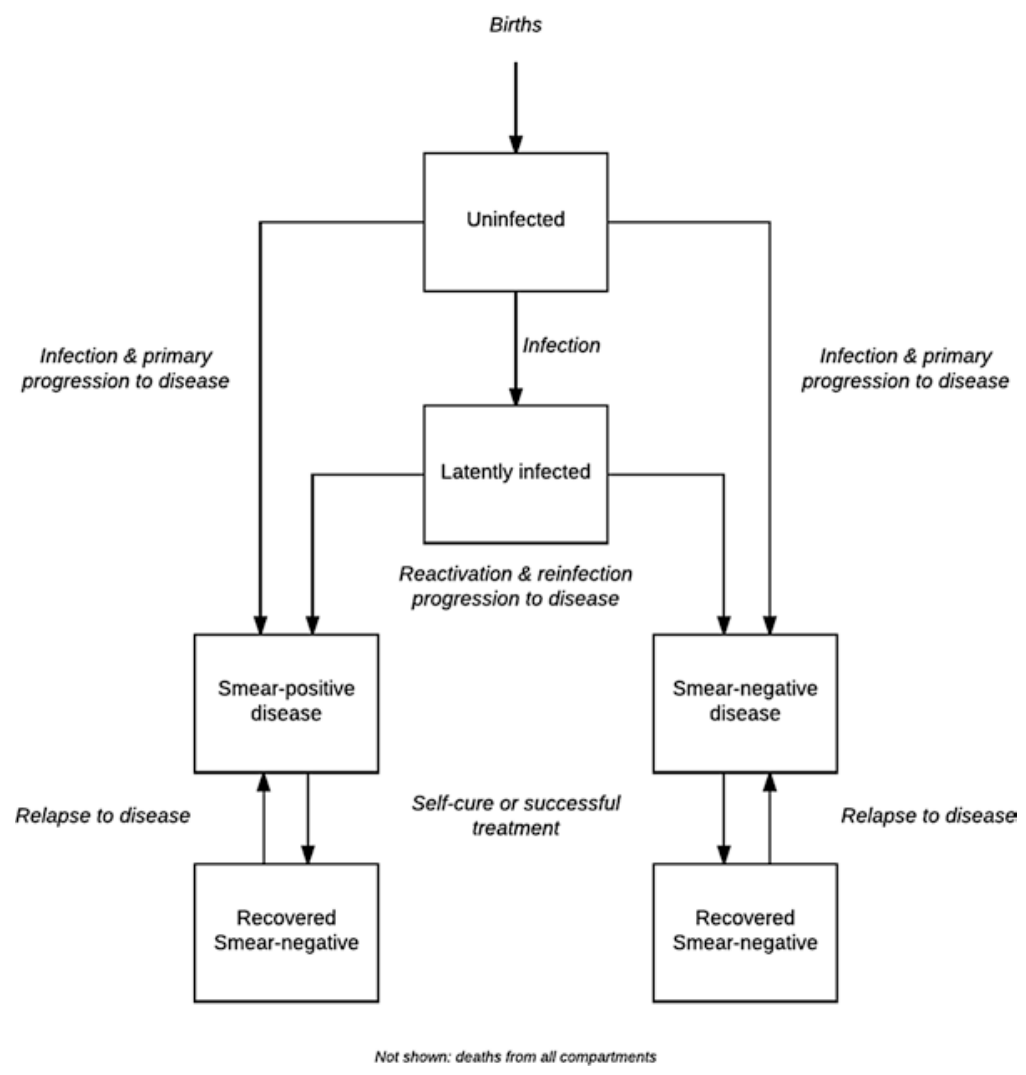

Fig. 2 A typical TB transmission model structure

in more realistically-specified particular populations, through to very detailed models tightly tied to the epidemiology and costs in specific settings, and aimed at policy evaluation and decision support. A list of modeling studies evaluating HIV and TB is given in Table 1 .

It should be noted that appropriate model structure is determined not only by the natural history or epidemiology, but also by the question at hand, and the published models vary widely in their structure. However, it is also the case that some models have appeared in the literature whose structure or parametrization means that they seriously misrepresent the natural history of TB. Menzies et al. [6] in a systematic review of TB modelling literature found that in $40 \%$ of published TB models there was an implied cumulative risk of progression to active disease that was substantially at odds with empirical data.

The model diagram in Fig. 2 maps precisely to simple 'compartmental models'. In these models, a set of mutually exclusive states that exhaustively classify individuals in the population are chosen. Each person in the population can then be thought of as being in one of these 'boxes' or 'compartments'. The model state at a given time is then given by the population number in each compartment and the population dynamics are determined by quantitative rules for the rates of flow 
Table 1 Summary of topics considered by TB modelling papers for high HIV prevalence settings

\begin{tabular}{|c|c|c|}
\hline $\begin{array}{l}\text { Focus on } \\
\text { intervention }\end{array}$ & Topic & First author, year and reference \\
\hline \multirow[t]{6}{*}{ No } & $\begin{array}{l}\text { HIV on TB } \\
\text { incidence }\end{array}$ & $\begin{array}{l}\text { Bermejo } 1992 \text { [67], Schulzer } 1992 \text { [68], Schulzer } 1994 \text { [70], } \\
\text { Dolin } 1994 \text { [69] }\end{array}$ \\
\hline & $\begin{array}{l}\text { TB dynamics with } \\
\text { HIV }\end{array}$ & $\begin{array}{l}\text { Kapitanov } 2015 \text { [74], Massad } 1993 \text { [71], Naresh } 2009 \text { [72], } \\
\text { Roeger } 2009 \text { [73] }\end{array}$ \\
\hline & $\begin{array}{l}\text { HIV on TB } \\
\text { evolution \& DR }\end{array}$ & Basu 2009 [76], Basu 2008 [75], Sergeev 2012 [77] \\
\hline & $\begin{array}{l}\text { HIV on TB } \\
\text { outbreaks }\end{array}$ & Murray 2002 [79], Porco 2001 [78], Pretorius 2011 [80] \\
\hline & $\begin{array}{l}\text { HIV on TB } \\
\text { transmission }\end{array}$ & $\begin{array}{l}\text { Andrews } 2014 \text { [84], Andrews } 2013 \text { [85], Dodd } 2016 \text { [86], } \\
\text { Escombe } 2008 \text { [81], Uys } 2011 \text { [83], Wood } 2010 \text { [82] }\end{array}$ \\
\hline & $\begin{array}{l}\text { Interpreting TB/ } \\
\text { HIV epidemiology }\end{array}$ & $\begin{array}{l}\text { Blaser } 2016 \text { [89], Hughes } 2006 \text { [87], Pretorius } 2014 \text { [90], } \\
\text { Sánchez } 2009 \text { [88] }\end{array}$ \\
\hline \multirow[t]{7}{*}{ Yes } & $\begin{array}{l}\text { TB/HIV dynamics } \\
\text { with interventions }\end{array}$ & $\begin{array}{l}\text { Agusto } 2014 \text { [91], Bacaër } 2008 \text { [94], Kaur } 2014 \text { [92], } \\
\text { Sharomi } 2008 \text { [93] }\end{array}$ \\
\hline & $\begin{array}{l}\text { Control of DR-TB } \\
\text { in TB/HIV } \\
\text { epidemics }\end{array}$ & Basu 2007 [63], Basu 2009 [96], Basu 2011 [97] \\
\hline & Preventive therapy & $\begin{array}{l}\text { Basu } 2009 \text { [101], Cohen } 2006 \text { [100], Guwatudde } 2004 \text { [99], } \\
\text { Heymann } 1993 \text { [98], Kunkel } 2016 \text { [103], Mills } 2011 \text { [102] }\end{array}$ \\
\hline & Case finding & $\begin{array}{l}\text { Azman } 2014 \text { [110], Baltussen } 2005 \text { [104], Dodd } 2011 \text { [42], } \\
\text { Dowdy } 2009 \text { [107], Dye } 1998 \text { [4], Laxminarayan } 2009 \text { [105], } \\
\text { Mellor } 2011 \text { [108], Sánchez } 2008 \text { [106], Yaesoubi } 2013 \text { [109] }\end{array}$ \\
\hline & Diagnostics & $\begin{array}{l}\text { Dowdy } 2014 \text { [62], Dowdy } 2006 \text { [111], Langley } 2012 \text { [114], } \\
\text { Langley } 2014 \text { [61], Lin } 2011 \text { [113], Menzies } 2012 \text { [112] }\end{array}$ \\
\hline & ART & $\begin{array}{l}\text { Bhunu } 2009 \text { [117], Dodd } 2013 \text { [118], Pretorius } 2014 \text { [119], } \\
\text { Williams } 2005 \text { [115], Williams } 2003 \text { [35], Williams } 2010 \text { [1] }\end{array}$ \\
\hline & $\begin{array}{l}\text { Combined } \\
\text { interventions }\end{array}$ & $\begin{array}{l}\text { Chindelevitch } 2015 \text { [122], Currie } 2005 \text { [120], Currie } 2003 \text { [121], } \\
\text { Gilbert } 2015 \text { [124], Gilbert } 2016 \text { [125], Houben } 2016 \text { [127], } \\
\text { Houben } 2016 \text { [129], Knight } 2015 \text { [123], Trauer } 2017 \text { [128] }\end{array}$ \\
\hline
\end{tabular}

Topics are in order of discussion in text. DR = drug resistant. The topic word 'dynamics' implies a more mathematically focussed study

between boxes, which may depend on the state of the system and on time. Certain flows are sometimes omitted from such model diagrams, e.g. the inflow of births into the uninfected class; the outflow of death from all states (at higher rates from disease states). Model diagrams, such as in Fig. 2 may suppress some detail for the sake of simplicity. An example is where models include a discrete number of age categories, each one of which would require a duplication of the infection/disease logic of Fig. 2. Model diagrams can also be used to describe more approximately the logic of modelling approaches that are not compartmental, e.g. individual- or agent-based models, which may include much finer subdivisions of the states and more flexible rules governing transition between states. Individual-based models (IBMs) explicitly track in silico representations of people, and can therefore deal with effects that are hard to represent when only dealing with groups of a certain type of person (e.g. a detailed dependence on the history that led to the current state rather than simply depending on the current state alone). 


\section{Modelling TB Natural History and Epidemiology}

\section{Infection and Progression}

One of the features of TB natural history that sets it aside from other infectious diseases and therefore distinguishes TB models from models of other infectious diseases is its long latent state and the complexity of this latent state's relationship to active TB disease. At birth, individuals are assumed to be uninfected and subject to infection with Mycobacterium tuberculosis (M. tuberculosis) with a force of infection (FOI) that depends on the prevalence of active TB disease at that time (see below). Individuals infected with $M$. tuberculosis for the first time are subject to an age-dependent risk of progressing to TB disease that decreases during childhood ages, remains low until adolescence and then increases as people reach adulthood [7]. This is normally represented as an elevated risk of 'primary progression' during the first $2-5$ years following infection, with a subsequent lower risk of 'endogenous reactivation' thereafter [8]. These rates of progression compete with mortality, such that only a minority of those infected will develop active disease as a result: the canonical rule-of-thumb is that once infected with TB people have a 10\% lifetime risk of disease [9], evenly divided between primary progression and endogenous reactivation, and more modern data broadly corroborate this picture [10]. In models, this is most often modelled as a certain fraction of infections progressing directly to active disease (an approximation to primary progression) and other individuals entering a latent category. Another modelling approach is to introduce two latent categories, with newly infected individuals moving initially into a fast-progressing latent category, before moving into a lower TB risk slow-latent category over a $2-5$ year time scale [11].

\section{Reinfection and Protection}

Another feature that is different from many other infectious diseases is the potential for those already carrying a latent $M$. tuberculosis infection (LTBI) to be reinfected. Evidence from modelling of population trends and more directly from cohorts of healthcare workers suggests that an existing latent infection conveys some protection against infection and progression to disease $[8,12,13]$. However, it is not possible to distinguish whether this protection applies against re-infection itself or progression following reinfection. If the latter, it is not clear whether there is any difference in the way this protection applies to progression in the initial years following reinfection or more distant re-activation. Re-infection is conventionally modelled as a partially effective 'degree' vaccine: i.e. infections in the latent (or slow-latent) category act like initial infections in the uninfected category, but occur at a lower rate with the FOI multiplied by some hazard ratio representing protection. This provides a contribution to incident TB disease from the latent category or via another route back into the fastlatent compartment. For modelling approaches with a single latent category, this approach covers either protection via reduced possibility of infection or via reduced progression. For modelling approaches with fast- and slow-latent compartments, this approach represents protection via reduced susceptibility to infection. 


\section{Infectiousness and Risks of Infection}

Upon development of active disease, many models assign individuals to either sputum smear-positive or smear-negative disease. This definition has been made operationally as it affects the changes of diagnosis by particular algorithms and represents a group historically emphasised in TB control approaches, but also because smearpositive TB is on average more infectious per unit time than smear-negative TB disease $[14,15]$. Some models introduce a low rate of progression from smearnegative to smear-positive disease [4], but this is poorly evidenced and considered small. While incipient smear-positive TB disease must at some point transition from smear-negative to smear-positive, the conventional modelling approach assumes this happens early on if at all, with around half of TB disease being smear-positive. It should be noted that new insights suggesting that the dichotomy between LTBI and clinical disease is an overly-simplistic representation of the dynamic spectrum existing in biological reality [16], and epidemiological data from prevalence surveys that have found substantial amounts of asymptomatic TB [17] have yet to be explored in terms of their implications for models. Typically, the force-of-infection in TB models is taken as being proportional to the per capita TB prevalence with a discounting factor for smear-negative TB prevalence. The coefficient of proportionality between per capita smear-positive TB prevalence and the force-of-infection is known in the epidemiological literature as Styblo's ratio: the number of infections per year generated by 1 smear-positive TB case. Historically this was estimated to be in the region of 10 infections per year, but may be closer to 6 infections per year in the modern era $[18,19]$. It is this dependence of force-of-infection on the current prevalence of active disease that makes a TB model 'dynamic' as opposed to 'static'. Changes in TB prevalence due to interventions here are allowed to accrue indirect benefits, influencing the future incidence of $\mathrm{TB}$ disease via a reduced force-of-infection.

\section{Mortality, Self-Cure and Detection}

The best data on the natural history of TB disease in the absence of treatment comes from the pre-chemotherapy literature [20]. A substantial proportion of those with TB disease would be expected to die: around $70 \%$ of those with smear-positive disease and around $30 \%$ of those with smear-negative disease, giving an average case-fatality ratio of around $50 \%$. The remaining cases are said to 'self-cure', represented as a return to latent infection compartments in TB models. The course of TB disease was found to run over an average of around 3 years (independent of smear-status), though data from the early 20th century suggest that some individuals could have active TB for over a decade [21].

The era of chemotherapy for TB has added a third class of outcome to TB disease: that a case is diagnosed and put onto treatment. This detection process is often abstracted into a single rate that captures both the efforts of a patient to seek care, and the sensitivity of the clinical algorithms used for diagnosis. Modelled in this way, rates of death, self-cure and detection can be considered as a competing hazard 
framework, with the rate representing detection competing against the rates of selfcure and death to determine the fraction of incident TB cases that are detected. In practice, this logic is usually inverted: information about the fraction of cases detected is used to determine the corresponding rate of detection.

\section{Treatment and Recovery}

Detection is usually modelled as synonymous with treatment initiation and those in the treatment category are normally assumed not to be infectious. This is based on the rapid decrease in both bacillary load and coughing frequency of TB patients on effective treatment [22, 23]. Exceptions include models that are used to investigate the significance of delays to treatment, and work including drug-resistant TB which may still be infectious on inappropriate treatment. Often, this low infectiousness on treatment and the small fraction of the population on TB treatment at any point mean that models do not include an explicit compartment for those on TB treatment. If an explicit TB treatment compartment is included it is normally modelled as a non-infectious compartment with a mean duration of 6 months (i.e. a total per capita exit rate of 2 per year).

The outcomes of treatment are often modelled as simply death or successful treatment, neglecting treatment failure or loss-to-follow-up (LTFU), with the total out rate split in proportion to the fraction experiencing each outcome. Surveillance data reported to WHO suggest that the treatment success for the 2015 cohort of new TB cases was 83\%, and 78\% in PLHIV [3]. Death, LTFU, and unevaluated outcomes heterogeneously account for most of the non-successful outcomes, with treatment failure rare in settings without very high levels of drug resistance. Globally, around $11 \%$ of TB treatments for new and relapse cases in PLHIV reported death as an outcome, compared with $4 \%$ of those without HIV [3]. Pre-treatment LTFU (i.e. LTFU between diagnosis and treatment initiation) is often incorporated into diagnostic rates, which may misrepresent the complexity in subsequent care-seeking by those who have at some point been diagnosed with TB.

Successful treatment outcomes and often self-cure of disease, are frequently modelled by a transition to a 'recovered' compartment. In contrast to the 'recovered' designation in most infectious disease models, this compartment is used to capture the elevated risk of recurrent $\mathrm{TB}$ disease in those previously treated for TB. TB treatment is not now thought to be sterilizing, and even after a documented cure, the risk of TB infection is greater than in people who have never developed the disease [24]. In many settings, approaching $10 \%$ of notified TB cases will be individuals who have been previously treated for TB, sometimes after LTFU, but more often after treatment completion. Recurrence is a complex phenomenon which includes disease following reinfection, reactivation, and mis-classified episodes of ongoing TB disease. Individuals with previous TB may be at elevated risk of TB infection and subsequent progression due to constant social or biological risk factors, or potentially due to changed risk factors resulting from their initial TB episode (lung scarring, exacerbated poverty). Many of these factors also increase the risk of reactivation disease from the original M. tuberculosis infection. 
This has important implications for TB control suggesting that previously treated patients should be followed-up regularly, possibly until the end of their lives. More recently a series of studies by Marx et al. have shown that, in the Western Cape Province of South Africa, previously treated patients have a relapse rate and a recurrence rate both of which are about 3000/100,000 in the first year after successful treatment which has important implications for the control of TB, especially in high burden settings [25, 26].

\section{Drug Resistance}

Recurrence may be linked with the local epidemiology of drug-resistant TB (DR$\mathrm{TB}$ ), which varies hugely around the globe, with proportions of multidrug-resistant or rifampicin-resistant TB (MDR/RR) among TB cases ranging from over 30\% in some former Soviet republics to less rates typically below 3\% in much of subSaharan Africa [3]. In all settings, the rates of drug resistance in cases with a history of TB treatment are higher than treatment-naive patients; treatment-naive patients are a barometer of transmitted resistance whereas cases previously treated reflect both acquired resistance during treatment and undiagnosed primary resistance that has been empirically determined to need second line treatment. MDR/RR TB is significant due to its worse outcomes and the much higher cost of treatment. Other resistance patterns, such as extensively drug-resistant (XDR) TB include resistance to common second-line compounds. Models including drug-resistant TB must replicate much of the structure to allow infection and transmission by strains of M. tuberculosis with different resistance patterns. The different outcomes, durations, relevant diagnostic algorithms and mechanisms for becoming a drug-resistant TB case must all be included in transmission models of DR-TB.

\section{Risk Factors Other Than HIV}

$\mathrm{HIV}$ is the single strongest risk factor for TB and the way its influence on the natural history of TB is typically incorporated in models of TB will be discussed in the following section. However, it is worth noting that other factors also influence the risk and characteristics of TB disease. Risks of progression to disease change rapidly during childhood, as does the spectrum of disease, requiring specific modelling approaches [27, 28]. The proportion of disease that is smear positive may increase through adult ages [8]. Biological and social risk factors mean that there are typically around twice as many TB cases in men as in women [29]. Diabetes mellitus is a moderate risk factor for TB incidence, but forecasts of increasing diabetes prevalence suggest a potentially important future role in global TB epidemiology [30]. Smoking, indoor air pollution, and silicosis are all risk factors that may make substantial contributions to TB incidence in some locales [31, 32]. A curious, but still unexplained, observation is the extremely strong association of TB with body-mass index (BMI) [33]. Across six longitudinal follow-up studies of navy recruits in the 
USA (2 studies), male smokers in Finland, Mass Radiography in Norway, Health Centres for the Elderly in Hong Kong and participants in an NHANES study in the USA, there was a $13.8 \% \pm 0.4 \%$ reduction in TB risk per unit increase in BMI over a range of BMI from $17 \mathrm{~kg} / \mathrm{m}^{2}$ to $34 \mathrm{~kg} / \mathrm{m}^{2}$, making BMI the single best predictor of an individual's relative risk of tuberculosis. Finally, through a variety of mechanisms, socioeconomic status has associations with TB incidence and outcomes and may well be a strong influence on global TB epidemiology during a period of rapid economic development in many high-TB prevalence regions [34].

\section{Modelling TB in Individuals with HIV}

\section{Data for Modelling}

In this section, we review the ways that the individual-level effects of HIV have been incorporated in TB models. It is worth noting that epidemiological evidence often needs particular interpretations, sometimes requiring additional assumptions, for use in mechanistic models. This is because mechanistic models are frequently more specific in their representations of causality than the statistical models conventionally used for analysing epidemiological data. Therefore, when incorporating effects which may act on one (or several) different pathways to effect, a more nuanced account of effect is required.

\section{Progression, Infection and Protection}

The main way TB models include the association between HIV and TB is through an increased rate of progression to TB disease for individuals with HIV infections. However, increased rates of progression could apply solely to 'primary progression' in the first couple of years following an infection, or to subsequent 'endogenous reactivation', or to both these processes (potentially in different ways). Data on TB incidence in cohorts of PLHIV suggest that the incidence rate ratio for developing TB increases immediately after infection by a factor of around 2, and remains higher, increasing as immunocompetence declines to over 30. Most models in the literature account for an increased rate of primary TB progression in PLHIV. Individual-based models and partial differential equation (PDE) models may include rates of progression to disease that vary continuously by time-sinceinfection. In these cases, without an enforced distinction between primary progression and endogenous reactivation, the most natural and commonly used approach is application of a single incidence rate ratio (IRR), which therefore elevates of both processes. The division between primary progression and endogenous reactivation, which is in any case a somewhat arbitrarily drawn distinction in practice, is further blurred in PLHIV when large IRRs may mean reactivation timescales are on a par 
with primary progression rates in HIV-uninfected individuals. Taken together, averaging across levels of immunocompetence, a rate of progression to TB disease in those with M. tuberculosis infections of $10 \%$ per year has been suggested among PLHIV, compared with a similar lifetime risk of progression among HIV-uninfected individuals [2].

Determining M. tuberculosis infection in PLHIV is particularly problematic, as sensitivity of both traditional tuberculin skin tests (TSTs) and newer interferon gamma release assays (IGRAs) is typically lower in this group due to immune dysfunction. It is therefore not possible to reliably determine whether HIV infection increases susceptibility to infection (as opposed to increasing progression to disease following infection). In models, it is usually assumed that HIV does not affect the risk of M. tuberculosis infection, and is a risk factor for higher TB incidence due to higher rates of progression to disease. The effect of HIV infection on the protection against reinfection disease conferred by LTBI is also poorly defined. Most models assume that HIV infection reduces or completely removes the protection against reinfection disease due to previous infection.

\section{Influence of CD4 Count}

There are a number of motivations for including a more detailed representation of HIV-related immunosuppression in TB models and for formulating this in terms of CD4 cell count. As mentioned above, there is more than an order of magnitude variation in IRR among PLHIV depending on their degree of immunosuppression. This is substantial enough to generate secular trends in the association between HIV and TB at a population level as HIV epidemics have aged. The count of CD4positive lymphocytes in peripheral whole blood samples (CD4 cell count) has been widely studied as a marker of immune suppression in PLHIV, and a guide for clinical decisions. Many guidelines on when to start ART have historically been based on CD4 counts, and there is much surveillance and survey data on CD4 counts at various stages of accessing care. This means that models including CD4 count could examine CD4-based ART policies and draw upon a wide range of data in their parametrization.

A number of analyses have identified a consistent relationship between CD4 count decline and increasing risk of TB incidence $[1,35,36]$. This relationship is exponential, that is a fixed decrement of 100 cells $/ \mathrm{mm}^{3} \mathrm{CD} 4$ count results in a 1.4fold factor increase in the IRR for TB [36]. Thus the roughly 20\% drop in CD4 cell count during the acute phase of HIV infection roughly doubles the risk of TB [35]. After the acute phase of HIV infection a person's CD4 cell count drops linearly (without ART) until death implying the risk of TB increases exponentially [35].

The IRR for TB given HIV varies widely on a population level: for example, the incidence rate ratio for TB in those with and without HIV ranges from 7.5 in Lesotho to 17.5 in Zimbabwe [37]. This may partly be due to the relative importance of socio-epidemiological factors, e.g. IRRs for TB given HIV are typically much lower 
for populations with generalized HIV epidemics than for populations with HIV epidemics driven by specific risk groups, whose members may be at elevated risk of TB due to other correlated risk factors amplified by assortative mixing. But this is likely only part of the story, and there is also substantial variation in baseline CD4-cell counts within and between populations [38]. Furthermore, for reasons still not understood, it seems that while the decline of CD4 cell counts is linear in time after HIV infection, the survival after infection is independent of the initial CD4 cell count, implying that people with a high CD4 cell count have a correspondingly faster rate of decline. However, this consistent exponential relationship with CD4 decrement across a number of settings and background TB risks means the strong biological component of increased risk from HIV can be separated from factors that do not depend on CD4 count.

Using CD4 count as a measure of immunosuppression for the IRR for TB has several advantages. Statistical analyses of data from the CASCADE cohorts provide models of CD4 count progression and their determinants, as well as HIV-related mortality [39, 40]. Modelling increases in TB risk through CD4 count means existing work on HIV natural history can be used directly in HIV/TB models, and allows TB models to be built on top of existing HIV model structures. There is an advantage in terms of parsimony: a single parameter can be used to capture the increased risk of TB in a variety of CD4 count compartments, by using an average implied IRR from the exponential model rather than separately assessing the risk for each CD4 category, and for models which continuously track time-since-HIV-infection or CD4 (IBMs, PDEs) this parametrization avoids binning into categories altogether. Incorporating several CD4 categories and parametrizing their TB risks via this exponential model is the approach most commonly used in applied models aimed at informing country policy. An example is the Spectrum HIV model developed by Avenir Health, which includes 7 CD4 categories, and the TB Impact Model and Estimates (TIME) modelling module that is part of the Spectrum tool and makes use of this underlying HIV model and the exponential model of TB risk with respect to CD4 count.

The natural history of HIV and TB are both different in children, who have much higher absolute CD4 cell counts that vary rapidly with age. The CD4 percentage is often used as an age-adjusted measure of immunosuppression. Systematic review and meta-analysis suggests that, as with adults, there is an exponential increase in the IRR for TB as CD4 percentage declines [41]. At a population level, an average over different levels of immunosuppression yields an IRR of around 8.

\section{TB Disease and Outcomes}

HIV infection shifts the spectrum of TB disease away from cavitary disease towards smear negative and extrapulmonary disease. One consequence of this is that the average HIV-positive TB case is usually assumed to be less infectious per unit time than the average HIV-negative TB case. This is often modelled as a lower proportion of disease that is classified as smear-positive. The natural history of TB/HIV without 
TB treatment is uncertain because HIV emerged after TB treatment was available, however, outcomes in individuals with DR-TB that was inappropriately treated, expert clinical opinion and other information do paint a consistent picture of a very much shorter duration of disease (of the order a few months rather than the few years of TB in HIV-negative individuals), with very limited or no self-cure (i.e. a case fatality rate of close to 100\%). Thus, although HIV increases the risk of TB by a factor of the order ten, TB disease progresses about ten times faster than in HIVpositive than in HIV-negative people. This means that while incidence may increase by up to ten times prevalence remains more or less constant as first shown by Corbett et al. [31]

Similarly, there is little evidence on TB case-detection rates in PLHIV. One could quantify rates of detection by HIV-status by examining HIV-stratified prevalence:notification ratios from using TB prevalence survey data, however TB prevalence surveys are not powered for conclusions by HIV status. The usual assumption in modelling is that TB has the same probability of being detected whether HIV positive or negative, although some studies have explored differential case detection [42]. This reflects much higher rates of care-seeking or screening for TB among PLHIV to compete with their higher rates of mortality. While evidence to directly inform this assumption is lacking, given population HIV prevalence and an IRR for TB among PLHIV, this assumption does generate HIV-prevalence among notified TB cases in keeping with observation.

TB treatment outcomes for PLHIV are somewhat worse than those of HIVnegative TB patients. In reality, outcomes are worse for more immunocompromised patients. All other characteristics of TB disease and detection are also likely to vary with level of immunosuppression, though this is rarely modelled. An exception is Williams et al. [1], which modelled the duration of untreated TB disease as being shorter for lower CD4 count.

\section{Effects of TB on HIV}

TB is one of the leading opportunistic infections listed as cause of death in PLHIV. Even if not fatal, it has been shown that TB disease can affect CD4 count in PLHIV and worsen HIV progression [43]. Finally, during some periods in some locales, TB disease has been a major route of HIV diagnosis and entry into HIV care. However, few studies have investigated the potential for bidirectional cross-talk between the HIV and TB epidemics. Most TB-focussed modelling exercises in high HIV-prevalence settings have used HIV incidence or prevalence as an input. This is no doubt partly because this requires dynamic HIV and TB models as well as specification of all their sources of interaction, which is challenging given the scarcity of data. It also potentially reflects the sense that the level of TB in a population may not greatly affect survival of untreated HIV; that if the primary cause of death at some level of immunosuppression had not been TB, it would have been something else. However, this assumption is unlikely to be true, especially at higher CD4 counts. 


\section{Modelling Interventions}

\section{Antiretroviral Therapy (ART)}

Coverage of ART among PLHIV globally has increased rapidly since 2004, and has potent benefits in reducing mortality almost to levels of HIV-negative individuals [44]. ART also benefits as a population-level HIV preventive tool [45], via reduced probability of transmission [46]. WHO guidelines now suggest initiation of ART in PLHIV regardless of CD4 count [47], though historically and still in some national guidelines, ART initiation is recommended only once CD4 count has fallen below some threshold.

Given its power to reduce HIV incidence and HIV-related mortality, ART provision has the potential to influence TB incidence through reductions in HIV prevalence in populations. However, ART also has direct individual level effects on TB.

Most notably, ART reduces the incidence of TB in adults, with a hazard ratio of around 0.35 across all CD4 counts [48], and to a similar degree in children [41]. The proportionate reduction in TB incidence may be higher for adults initiated at the lowest CD4 counts, whose rates of TB would otherwise be highest [48]. The protection from TB appears to increase over the first few years on ART in line with markers of immune status [49, 50], and somewhat more rapidly in children [41]. However, TB rates in PLHIV on ART compared to HIV-uninfected adults appear to remain at least 4 fold higher, even after 5 years or more on ART [51].

Being on ART may shift the spectrum of TB disease back in the direction of the HIV-negative spectrum, likely increasing infectiousness, decreasing case fatality rate, and potentially allowing some degree of protection stemming from LTBI and lengthening disease duration. Most models have treated TB disease in PLHIV on ART as very similar or identical to TB disease in PLHIV not on ART, and transmission models have not considered unmasking, immune reconstitution inflammatory syndrome (IRIS), or drug-drug interactions [52]. TB treatment outcomes are improved in PLHIV by being on ART, and are comparable with those for HIV-uninfected TB patients [53].

However, regular attendance of ART clinics may well also have implications for TB detection rates. Aside from acting as a marker for those able and motivated to access care, it also signals a heightened index of suspicion for TB among both patients and clinicians. Conversely, ART clinics have also been suggested as the source of a potentially heightened exposure to TB: the Tugela Ferry outbreak of XDR TB centred on an ART clinic, and high rates of active TB have been found among ART clinic attendees [54]. These features, which may have national-level implications in high HIV burden settings, remain largely unexplored in modelling studies.

\section{Isoniazid Preventive Therapy (IPT)}

Isoniazid preventive therapy (IPT) to lower the rate of progression to TB disease is currently recommended for 36 months in PLHIV [55]. Shorter courses of IPT have been shown to lower TB incidence by around $60 \%$ in PLHIV with a positive skin 
test [56], and data comparing TB incidence for individuals on ART with that in those on both ART and IPT [57], and comparing TB incidence or mortality reductions from IPT in cohorts with or without ART [58] suggest IPT has an incremental benefit while taking ART.

While IPT has sometimes been regarded historically as protecting by clearing a latent infection, trial data evaluating extended durations of IPT in PLHIV (including those on ART) [59] and mathematical modelling fitting to data from a set of IPT studies in PLHIV not on ART with post-prophylaxis follow-up [60] both suggest that IPT is unlikely to clear M. tuberculosis infection in most PLHIV. This means that the risk of TB incidence after treatment cessation is likely to return to its level before prophylaxis, and has implications for the way IPT should be modelled in PLHIV (i.e. as a temporary reduction in risk). Houben et al.'s modelling also suggested a higher curative effect of rifamycin-containing prophylactic regimens among PLHIV [60], tallying with biological understanding of mechanism.

\section{Improved TB Detection}

Transmission models that have focussed on evaluating diagnostic algorithms within clinics tend to have relatively simple representations of either HIV or of the diagnosis process, with the focus of detail being on the complementary aspect. Most frequently, the effects of changed algorithm are reflected in different rates into treatment states in a compartmental model. This may neglect the true complexity of repeated attempts to obtain a diagnosis, diagnosis of previously treated TB and similar details. Sometimes these changes in transmission parameters may be derived from more detailed operational models of diagnostic procedures [61], or calculated by approximations to decision trees giving mean sensitivity, specificity and delay for patient types represented in the transmission model [62]. Applying simple changes in a detection rate (e.g. in proportion to changes in test sensitivity) without considering additional compartments representing pre-care-seeking infectiousness may exaggerate the potential for improvements in passive detection to affect transmission.

More active approaches to case-detection are variously-termed and understood (e.g. active case-finding, enhanced case-finding, systematic screening), and have been the subject of transmission modelling analyses in high-HIV burden settings (intensified case-finding, conventionally referring to screening among PLHIV, seems to have received less attention from transmission modellers). Periodic rounds of active case-finding have been modelled as mechanistically removing a proportion of prevalent cases each round. Often, active or enhanced case-finding is conceptualized more nebulously as improved case-detection from direct efforts and potentially improved community awareness, and represented as increases in detection rate parameters. Understanding when transmission occurs on average with respect to care-seeking and symptoms is a crucial uncertainty that affects the relative impact of active case-finding interventions. 
Household contact-tracing has also been considered in these settings, typically using individual-based models that can naturally represent households of index cases.

\section{Infection Control}

Improvements in ventilation for preventing nosocomial transmission of TB have been modelled by using the Wells-Riley equation and its variants, which quantify the reduction in infectiousness achieved by changes in ventilation rates [63]. More sophisticated specific approaches have been used to model the impact on ventilation of specific changes to buildings [64], and of upper room ultraviolet germicidal irradiation [65], but this has yet to be combined with transmission modelling in highHIV burden settings.

\section{Population-Level Impacts}

In this section, we review the mathematical modelling literature that has included explicit representation of TB transmission and of the effect of HIV on TB epidemiology. We have divided the literature into work describing the influence of HIV on TB epidemics that does not evaluate the impact of interventions, and work whose main focus is in evaluating single or multiple interventions that reduce TB burden. In focussing only on modelling work that includes transmission, we have excluded a substantial body of work evaluating the individual-level impact and costeffectiveness of TB interventions among PLHIV (e.g. some of the work on IPT, diagnostic approaches). We have also included some work focussed on modelling the transmission process in high-HIV burden settings (e.g. using Wells-Riley approaches), but which does not use a transmission model (in the usual sense) to propagate the consequences of this transmission. In writing this section, we have drawn on a systematic review of the TB/HIV modelling literature up to 2012 [66], and updated it using the same search terms. See Fig. 3 for temporal trends in number and topic of these publications.

There are several key points that determine the way in which one models HIVrelated TB. First of all, standard TB models for modelling HIV-negative TB can be used even in the presence of a substantial HIV epidemic TB because HIV has a much larger impact on TB incidence than prevalence, and therefore transmission. Secondly, the time-scales for HIV are of the order of years but for TB are of the order of decades which allows the separation of the models. Given a model of TB that fits the data before the epidemic of HIV starts, simple approaches need only a few additional parameters to include TB/HIV: a parameter (around 2), which gives the increase in the risk of TB immediately after infection with HIV; a parameter which gives the relative risk of TB when HIV-positive people are on ART; and a 


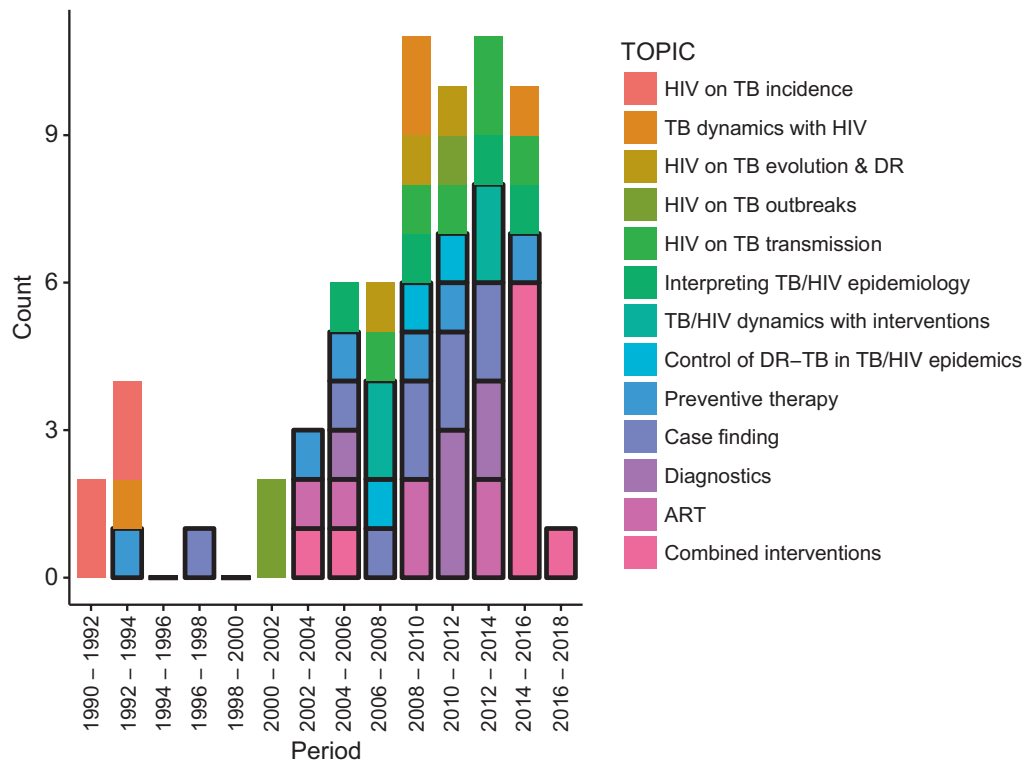

Fig. 3 Temporal evolution of topics considered by TB modelling papers for high HIV prevalence settings. Bars with solid borders represent studies with a focus on modelling interventions. Bar colours are in the same order as in the legend. DR = drug resistant. The topic word 'dynamics' implies a more mathematically focussed study

parameter specifying the exponential rate of increase in TB incidence as people progress to successive clinical stages of HIV, which may differ between populations to account for the substantial variation in the incidence rate ratio in different populations. A detailed discussion of these observations is given in the supporting information of Williams et al. [37]

\section{Population-Level Impact of HIV on TB Epidemics}

The earliest papers using modelling tools to understand TB/HIV epidemics focussed on drawing attention to the threat posed by HIV to TB control and making estimates of the increases in TB incidence due to HIV. Bermejo et al. [67] in 1992 used a simple mathematical model and ecological data on associations between HIV and TB to conclude that TB incidence would double going forward when adult HIV prevalence hit $13 \%$. Schulzer et al. [68] in the same year concluded that the TB incidence in 15-45 year olds was likely to increase by factors of between 4 and 12 by the year 2000 compared to 1980, depending on the baseline annual risks of M. tuberculosis infection, and projected HIV prevalence. Dolin et al. [69] in 1994 generated TB burden forecasts for 1990-1999, projecting 88 million new TB cases for this period with $10 \%$ of them HIV associated. The same year, Schulzer et al. [70] introduced a more sophisticated actuarial back calculation approach, and projected 
2-6 fold increases in TB incidence in sub-Saharan African populations by 2000 compared with 1980. These predicted increases are in line with those observed over the period. Later, in their 2003 review of global TB/HIV epidemiology, Corbett et al. [2] used simple mathematical tools to interpret data that were subsequently very influential in the applied TB/HIV modelling literature.

The first article to introduce a traditional compartmental transmission model based on ordinary differential equations (ODEs) was Massad et al. [71] in 1993. This work was in a dynamical systems and mathematical biology tradition, but did conclude that the influence of HIV on TB at a population level was much stronger than the other way around. Other authors followed in this vein, exploring the stability properties of systems of ODEs motivated as TB/HIV models, but with a focus more on mapping out possible behaviours of the abstract dynamical systems than closely tying the models to a real setting or settings [71-73]. (Naresh et al. [72] is notable and unusual for considering the population-level impact of TB on HIV, however.) Kapitanov [74] introduces and analyses a PDE model, separately including time-since-infection for HIV and M. tuberculosis.

Authors beginning with Basu et al. [75] in 2008 have followed a population biology tradition and focussed on the potential impact of HIV for the evolution of TB, especially DR-TB. This first article considered cross-immunity between TB strains in a multi-strain HIV/TB model, and highlighted the potential for HIV to promote the emergence of DR-TB at a population-level by compensating for reduced fitness in DR-TB strains. In a later paper Basu et al. [76] consider the evolution of TB virulence, again concluding that HIV may facilitate increased virulence. Sergeev et al. [77] agree that HIV can facilitate DR-TB epidemics, but explore the dynamics of the relationship between HIV and DR-TB, predicting a lower proportion of DR-TB among PLHIV early on in DR-TB epidemics due to enhanced reactivation of older strains in this group.

Models have also been used to explore the influence of HIV on stochastic aspects of TB incidence in small communities or lower burden settings. In 2001, Porco et al. [78] used discrete event simulation (DES) to study the influence of HIV on the frequency and severity of TB outbreaks. Murray et al. [79] included HIV in their analysis of the determinants of molecular cluster size. More recently, Pretorius et al. [80] used a van Kampen expansion to explore the effects of stochasticity in a TB model applied to a medium size high-HIV community, and examined the temporal correlations for active TB cases.

Another strand of work has made use of the classical Wells-Riley model of indoor transmission and ventilation to interpret experimental work on the relative infectiousness of TB in PLHIV [81], to consider the role of household transmission in generating observed acute respiratory illnesses (ARI) in South Africa [82], and arguing implications at the population-level and a necessity for more-than-proportionately intense interventions in intense transmission settings (again focussed on South Africa) [83]. Andrews et al. [84] have modelled the implications of data on social contact patterns, and $\mathrm{CO}_{2}$-derived measures of ventilation, for the location of TB transmission in South Africa, singling out the role of public transport in another study [85]. Dodd et al. [86] also make use of social contact data (from Zambia and South Africa), 
together with data on TB prevalence and infection rates, to model the age- and sexspecific rates of TB infection, arguing ARI measured in children under-estimates that experienced by adults.

Lastly, transmission models have been used to interpret the patterns and trends in epidemiologic data from high-HIV burden settings. In 2006, Hughes et al. [87] used a DES model calibrated to Zimbabwe, to understand the impact of HIV on the TB epidemic there. In an intriguing article, Sanchez et al. [88] fitted a difference equation model of HIV and TB in sub-Saharan African settings, notably Kenya, highlighting the difficulties of matching the observed trends and exploring potential reasons for discrepancies. Blaser et al. [89] developed an age-structured TB/HIV model calibrated to Cape Town in order to understand the epidemiology. They conclude that protection from LTBI and higher progression rates in previously treated individuals are key in being able to reproduce age-patterns of TB. Finally, Pretorius et al. [90] developed a regression methodology to model the influence of population CD4 changes on TB incidence and is used to disaggregate TB incidence by CD4 stratum in the TIME model (part of the Spectrum model suite for country-level estimation of TB/HIV mortality burden).

\section{Population-Level Impact of Interventions}

A number of studies considering the impact of interventions on TB/HIV have appeared in the mathematical literature. Agusto et al. [91] considered optimal control theory applied to a two-(TB)strain TB/HIV ODE model. Kaur et al. [92] studied an ODE TB/HIV model that includes abstractly defined screening and treatment interventions from a dynamical systems perspective. Sharomi et al. [93] also applied dynamical systems analysis to a TB/HIV ODE model, but with more explicit representation of intervention strategies including ART. The study of Bacaër et al. [94] is more realistic, and calibrates an TB/HIV ODE model to a specific (South African) setting. Interventions for HIV including ART and condom use promotion, as well as IPT for TB are considered: ART was predicted to strongly reduce TB notifications.

Motivated by the 2006 outbreak of XDR-TB in Tugela Ferry, South Africa, in which ART clinics appeared to play a key role, Basu et al. [63] use an TB/HIV ODE model including drug-resistant TB to model intervention strategies to reduce nosocomial transmission of TB, concluding that a combination of strategies could prevent around half of XDR cases. It was shown that the outbreak of XDR-TB in Tugela Ferry was largely due to nosocomial transmission resulting from poor infection control in a clinic with very high rates of both HIV and TB [95]. Basu et al. [96] went on to consider this with a stochastic model appropriate to the relatively small numbers of this outbreak situation, concluding that community-based interventions would be needed to curtail the outbreak. Later Basu et al. [97] considered the role of institutions such as prisons in amplifying TB epidemics, and argue that reducing the inflow to these institutions would have impacts on both TB incidence and the propagation of drug-resistant TB. 
A number of studies have considered IPT. In keeping with our scope, we discuss only those including transmission; it should be noted that IPT in PLHIV has been the focus of a similar number of static cost-effectiveness modelling analyses. In 1993, Heyman [98] considered the impact of HIV on TB in HIV-hyperendemic settings with low TB treatment coverage, and the impact of preventive therapy (assumed curative) aimed at PLHIV, suggesting a large reduction in prevalence could result over a decade. In 2004, Guwatudde et al. [99] developed an TB/HIV ODE model and concluded that IPT for PLHIV would have a limited impact on the TB epidemics of sub-Saharan Africa. In 2006, Cohen et al. [100] also modelled IPT for PLHIV in sub-Saharan Africa and found a greater potential for this intervention, with up to around $20 \%$ of cumulative HIV deaths preventable through this strategy over a 5 -year period. However, they also found a potential for increases in drug-resistant $\mathrm{TB}$, and argued that IPT should therefore be coupled with improved diagnostic and treatment options. Basu et al. [101] specifically consider IPT delivered through ART clinics in Botswana, found that increases in resistance were small and more than counterbalanced by reduced TB incidence and mortality. Mills et al. [102] introduced a dual-network model - one network for contacts relevant to HIV transmission, a second network with contacts relevant to TB transmission - and investigated the role of correlations between these structures in generating heterogeneity in the impact of IPT. IPT was found to be effective at a population-level, but networks with clustering of HIV and TB transmission in intense foci had reduced local impact from IPT due to high rates of TB reinfection. Kunkel et al. [103] calibrated a multistrain TB model with HIV to data from Botswana and consider the health benefits and resistance dynamics resulting from continuous IPT for PLHIV. They find health benefits outweigh concerns around increased resistance, so long as sufficient control is maintained for the overall TB epidemic.

Various studies have used modelling to evaluate TB case finding in high-HIV burden settings, including both improvements in passive case detection and cure under the DOTS strategy, and through active case finding. The early paper of Dye et al. [4] projected the global and regional impact of the DOTS strategy (focussed on case detection and treatment success) and included HIV. They concluded that DOTS has a greater impact on mortality than incidence and that this difference is heightened in settings where HIV is prevalent. Baltussen et al. [104] built on the work of Dye et al. [4] to evaluate the cost-effectiveness of DOTS and DOTS-plus on a regional and global level. Laxminarayan et al. [105] undertook a later global country-level economic evaluation of sustaining DOTS, finding that the benefits exceeded costs in all of the (then-designated) 22 high TB-burden countries. Sanchez et al. [106] considered increased detection as well as decreased LTFU and higher cure of shorter regimens for PLHIV in a transmission model calibrated to the epidemiology of Kenya, projecting a $20 \%$ reduction in TB incidence and mortality by 2030 for these interventions in combination. Dowdy et al. [107] considered the effects of improvements in case detection on TB incidence, finding that declines in TB incidence reduce over time since an improvement in case-detection. Dodd et al. [42] considered what features affect periodic active case finding for TB in high-HIV burden settings using a PDE model, and allowing different baseline detection characteristics for HIV- 
positive and HIV-negative TB cases. For a given case-finding round efficacy, the impact is higher in settings where a higher proportion of TB incidence is due to recent infection. Mellor et al. [108] used an individual-based household-structured model based on data from Zimbabwe to consider the impact of different household contact tracing (HHCT) interventions to screen for TB disease and offer IPT, and found HHCT for late-stage HIV-positive individuals was particularly effective in reducing mortality. Yaesoubi et al. [109] considered optimal dynamic rules for switching on and off active or enhanced TB case finding efforts in addition to passive case finding given a decision rule based on a policy-make willingness-to-pay threshold, concluding that dynamic strategies are more efficient and therefore potentially more feasible and sustainable in practice than fixed case-finding strategies. Lastly, Azman et al. [110] consider the cost-effectiveness of active case-finding in three countries, including South Africa, concluding that the modelled intervention would be cost-effective in South Africa, and that active case-finding strategies have greater cost-effectiveness when considered over longer time horizons.

The impact of different diagnostic strategies in HIV-driven TB epidemics has also been considered. Dowdy et al. [111] in 2006 considered the impact of rapid molecular testing or culture compared to active case-finding or wider ART use; finding that while improved diagnostic strategies only moderately reduce TB incidence (by around 10\%), their effect on TB mortality is larger (around 20\%). Dowdy et al. [62] introduce a transmission modelling framework focussed on diagnostic strategies, which did include a simple aggregate representation of HIV. Menzies et al. [112] undertook a rigorous economic evaluation of Xpert MTB/RIF in five southern African countries, using a TB transmission model that included the effects of HIV. This analysis too found limited impacts on TB incidence, but benefits in terms of TB mortality and morbidity. Longer time horizons favoured the intervention because they allowed indirect benefits to accrue. Another stream of modelling work has sought to integrate operational research models of practical implementation details and workflows with transmission models for evaluating new diagnostics in high TB (and high HIV) burden settings [61, 113, 114]. For example, Langley et al. [61] also evaluated Xpert (together with fluorescence LED microscopy) in Tanzania, finding Xpert to have the greatest cost and impact, and allowing estimation of quantities such as reductions in patient visits to health facilities.

ART for HIV is one of the key interventions whose impact on TB in high-HIV burden settings has been considered. In 2003, Williams et al. [35] developed a cohort model and brought evidence together on the efficacy of ART for preventing TB and the incidence rate ratio for TB at different CD4 counts, highlighting the importance of starting ART early and achieving high coverage and compliance in reducing cumulative TB incidence. In Williams et al. [115], a transmission model with a 4-stage HIV structure was used to project the impact of HIV on the Indian TB epidemic, finding that continued progress with TB control activities should contain the impact of HIV, but recommending ART to PLHIV who develop TB. The evidence on the individual impact of $\mathrm{CD} 4$ cell count and ART on TB risk was updated in Williams et al. [1] and incorporated in a TB/HIV model applied to 9 sub-Saharan African countries to investigate the impact of a 'universal test-and-treat' (UTT) HIV control 
strategy on TB. UTT comprises frequent HIV testing at high coverage followed by immediate ART initiation, and has been projected to have the potential to reduce HIV incidence by reducing the infectiousness of PLHIV [116]. This study included the impact of aggressive ART beginning in 2010 on HIV incidence, and predicted a potential reduction in TB/HIV by more than $50 \%$ by 2015 ; strategies achieving ART initiation within 2 years of HIV infection led to more than $95 \%$ reductions in TB/ HIV by 2050 . The approaches to modelling the relationship between TB and HIV introduced in these papers, confirmed by more recent evidence synthesis [36], have been very influential on other modelling approaches to TB/HIV.

Some work on the impact of ART on TB has included work in a mathematical tradition such as Bhunu et al. [117], who pursue a dynamical systems analysis of an TB/HIV model including ART, and Bacaër et al. [94], discussed above. Dodd et al. [118] caution that the still-high relative risks of TB on ART and the longer lifeexpectancies imply that long-term reduction in TB from ART must be mediated by reductions in HIV incidence or background population risks of TB. Pretorius et al. [119] report results from three independent models assessing the impact of ART policy changes in South Africa over the period 2014-2033, finding expanded coverage and universal eligibility could reduce cumulative TB incidence and mortality by around $20 \%$ and $30 \%$, respectively over this period, with one TB case averted for every person-decade or so on ART.

In addition to the studies focussed on ART discussed here, many of the models considering combinations of interventions (discussed below) have included ART as a component of their policy options, alongside other interventions. Currie et al. [120] consider three interventions to prevent TB in Uganda, Kenya and South Africa - namely IPT, ART, and reduced HIV transmission - with curative interventions (improved TB case detection and treatment success). They found the largest impacts on TB from curative interventions, but cautioned that alone they were able to contain but not reverse TB epidemics. Currie et al. [121] built on this work to consider the cost-effectiveness of interventions in Kenya, finding that improvements in TB detection and cure were extremely cost-effective, but noting that ART policies had the largest potential for general health gains, while being the most expensive and relatively expensive measured as a TB-prevention strategy. Chindelevitch et al. [122] compared wider ART provision and improvements to TB control for improving TB control in South Africa, finding that expanded coverage of the TB programme had the greatest potential for impact on TB, but suggesting a potential $22 \%$ reduction in cumulative TB incidence over 5 years from expanded ART eligibility. Knight et al. [123] also considered South Africa, using an individualbased model to evaluate portfolios of interventions including expanded ART, longduration IPT, and active case finding for achieving the national targets to find that the most aggressive combination considered could result in TB incidence and mortality rates that were $70 \%$ and $86 \%$ lower than those in 2012 . The most effective single intervention was general-population active case finding for TB, whereas improvements in ART had more modest impacts: UTT alone generated around a $20 \%$ reduction in TB incidence. Gilbert et al. [124] considered a combination of intervention strategies in rural South African settings, including ART, IPT, and a 
community-based integrated intensified case finding strategy for HIV and TB. The intensified case finding was found to be the single most effective intervention for TB incidence, with around a $25 \%$ reduction over 10 years and a comparable impact on combined TB and HIV mortality as expanded ART policies. Gilbert et al. [125] built on this to evaluate the projected cost-effectiveness of their intensified case finding strategy, finding it cost-effective in rural South Africa.

A number of studies recently have gathered multiple modelling teams together to address the same question. The study of Pretorius et al. [119], discussed above, was a result from a multi-modelling study focussed on ART policies for HIV [126]. In 2016, Houben et al. [127] undertook a study with 11 different models focussed on 3 countries to assess approaches to meeting the End TB strategy goals. South Africa was one of the countries considered by the 8 models including the necessary HIV structure for this context, and it was found that a combination of continuous IPT for PLHIV on ART, TB screening at health facilities, and improved TB care could reduce TB incidence and mortality in 2025 by $55 \%$ and $72 \%$, respectively, compared with 2015 .

Finally, a number of country-level TB modelling tools are being developed to undertake national policy analyses, often for informing applications for support from the Global Fund to Fight AIDS, Malaria and Tuberculosis [128, 129]. For example, the TIME model is built into the widely-used Spectrum suite of models, which have a heavy emphasis on HIV policy, and has been applied to a number of high-HIV burden countries. Most of the outputs from this work have yet to be described in peer-reviewed literature.

\section{Discussion}

\section{Uses and Achievements of Modelling}

One of the key uses of TB/HIV epidemic modelling has been as a tool to understand the drivers of epidemiological patterns and project the effects of HIV on TB epidemics. Some of the early studies were based on simple models but provided important insights. The first projections of the effect of HIV on TB incidence proved broadly correct, and the understanding that the association between TB and HIV is a dynamic one, driven by mean population immune status is also borne out by observation. Early fears that TB in PLHIV might be more infectious than in HIVuninfected individuals proved wrong, and together with the much shorter duration of TB/HIV compared with TB in HIV-uninfected individuals means that even in settings where the majority of incident TB is HIV-associated, it will still often be the case that TB in the HIV-negative population drives the majority of transmission. This implies that efforts to strengthen TB control in these settings must also encompass the HIV-negative population, while recognising the special challenges of TB/HIV from increased risks of mortality and different clinical presentation.

Modelling work has also established the potential for HIV to have a facilitating effect on the development and establishment of DR-TB in populations; the impaired 
host immune responses compensating for reductions in pathogen transmission fitness or virulence. Modelling has also explored the role of ART clinics in facilitating $\mathrm{TB}$, and particular DR-TB transmission in the wider community.

Understanding the impact of new or existing interventions and prioritizing their future development has also been an important area where models have been useful. The application of modelling to IPT in PLHIV is a good example of understanding existing interventions: data from randomized studies are often analyzed at an individual level; sometimes transmission effects and the potential for reinfection with M. tuberculosis may have a bearing on the interpretation of results from individuallevel trials. Modelling has been particularly influential in investigating the potential for impact from innovative strategies that have not yet been trialled at population level. HIV modelling provided some of the evidence supporting the policy shifts to universal ART provision, and related TB modelling has studied the potential for additional benefits from these policies effects on the TB epidemic. Modelling studies are able to explore more interventions, intervention variants, and combinations of interventions than would ever be possible to trial in empirical studies, thus helping to prioritize and design interventions to take forward for empirical evaluation.

Models of TB/HIV are also increasingly being used to guide policy and investment decisions, including both epidemiological impact from changes in TB transmission and the costs and benefits needed to generate health economic evaluations of policy options. Multiple models have been applied to harmonized scenarios in specific settings, particularly with the aim of establishing a consensus about ingredients needed to move towards TB control targets using current tools. Practical use of country-level modelling of TB in high-HIV burden settings to support policy design and applications for donor funding has also been increasing. This is an important area of work that is as yet less documented in the academic literature.

\section{Future Directions}

For a combination of serious conditions that afflicts in the region of 1 million people in the world each year, TB/HIV has not received the attention from epidemiological modellers that it should have. This is perhaps due to the genuine complexity of dealing of the interactions between two conditions which each have their own complexities in terms of epidemiology, natural history, and control policies. However, it means that there are many areas where modelling could be usefully applied going forwards.

More could be done to use modelling as a tool to understand epidemiology and the performance of control efforts. ART has scaled up hugely over the last decade; modelling using routine and study data could be used more to understand the long-term impact of ART on TB/HIV epidemiology and chart its likely future course. This may require increasing attention to interactions with other societal features that are rapidly changing in high-HIV and TB burden settings, such as urbanization, improvements in nutritional status, and increasing prevalence of diabetes mellitus. Modelling has been used in conjunction with social contact data and 
ventilation data to assess likely contributions of particular locales to TB transmission, and the impact of infection control interventions aimed at particular hot-spots; more work in this area would help improve our knowledge of an important but poorly-understood aspect of TB epidemiology and potentially suggest relatively easily-implemented interventions.

Finally, some workers have begun to include the operational details of health systems and care provision in models, and there is an increasing demand for these details that mesh with the level where design decisions are taken and where costsaccrue. Developing such models presents challenges in terms of the range of expertise required by the teams involved, but also in terms of modelling techniques for combining models that may have very different emphases and ideal approaches to implementation separately. Increasing availability of electronic health records may also make easier the job of parametrizing the health system aspects of such models.

\section{Conclusion}

Population-level modelling of TB in high-HIV burden settings has helped bring together a wealth of understanding around TB/HIV natural history, epidemiology and interventions to provide important insights into the implications for TB transmission and control. Increasingly, models are being used to guide policy and investment decisions at a country and supranational level. TB/HIV remains a substantial global health concern, and important questions and challenges remain to be addressed by transmission modelling.

Acknowledgments This publication was produced by TREATS which is part of the EDCTP2 programme supported by the European Union (grant number RIA2016S- 1632-TREATS). The views and opinions of authors expressed herein do not necessarily state or reflect those of EDCTP.

\section{References}

1. Williams BG, Granich R, De Cock KM, Glaziou P, Sharma A, Dye C (2010) Antiretroviral therapy for tuberculosis control in nine African countries. Proc Natl Acad Sci U S A 107:19485-19489

2. Corbett EL, Watt CJ, Walker $\mathrm{N}$ et al (2003) The growing burden of tuberculosis. Arch Intern Med 163:1009

3. World Health Organisation (2017) Global tuberculosis report 2017

4. Dye C, Garnett GP, Sleeman K, Williams BG (1998) Prospects for worldwide tuberculosis control under the WHO DOTS strategy. Directly observed short-course therapy. Lancet 352:1886-1891

5. Dye C. The population biology of tuberculosis. 2017.

6. Menzies NA, Wolf E, Connors D, Cohen T, Hill AN, Yaesoubi R, Galer K, White PJ, Abubakar I, Salomon JA (2018) Progression from latent infection to active disease in dynamic TB transmission models: a systematic review. Lancet Infect Dis 18(8):e228-e238 
7. Marais BJ, Gie RP, Schaaf HS et al (2004) The natural history of childhood intra-thoracic tuberculosis: a critical review of literature from the pre-chemotherapy era. Int J Tuberc Lung Dis 8:392-402

8. Vynnycky E, Fine PE (1997) The natural history of tuberculosis: the implications of agedependent risks of disease and the role of reinfection. Epidemiol Infect 119:183-201

9. Comstock GW, Livesay VT, Woolpert SF (1974) The prognosis of a positive tuberculin reaction in childhood and adolescence. Am J Epidemiol 99:131-138

10. Sloot R, van der Loeff MF S, Kouw PM, Borgdorff MW (2014) Risk of tuberculosis after recent exposure. A 10-year follow-up study of contacts in Amsterdam. Am J Respir Crit Care Med 190:1044-1052

11. Ragonnet R, Trauer JM, Scott N, Meehan MT, Denholm JT, McBryde ES. Optimally capturing latency dynamics in models of tuberculosis transmission. Epidemics 2017. https://doi. org/10.1016/j.epidem.2017.06.002

12. Andrews JR, Noubary F, Walensky RP, Cerda R, Losina E, Horsburgh CR (2012) Risk of progression to active tuberculosis following reinfection with Mycobacterium tuberculosis. Clin Infect Dis 54:784-791

13. Sutherland I, Svandova E, Radhakrishna S (1976) Alternative models for the development of tuberculosis disease following infection with tubercle bacilli. Bull Int Union Tuberc 51:171-179

14. Tostmann A, Kik SV, Kalisvaart NA et al (2008) Tuberculosis transmission by patients with smear-negative pulmonary tuberculosis in a large cohort in The Netherlands. Clin Infect Dis 47:1135-1142

15. Hernandez-Garduno E (2004) Transmission of tuberculosis from smear negative patients: a molecular epidemiology study. Thorax 59:286-290

16. Esmail H, Barry CE 3rd, Young DB, Wilkinson RJ (2014) The ongoing challenge of latent tuberculosis. Philos Trans R Soc Lond Ser B Biol Sci 369:20130437

17. Onozaki I, Law I, Sismanidis C, Zignol M, Glaziou P, Floyd K (2015) National tuberculosis prevalence surveys in Asia, 1990-2012: an overview of results and lessons learned. Trop Med Int Health 20:1128-1145

18. Trunz BB, Bourdin Trunz B, Fine P, Dye C (2006) Effect of BCG vaccination on childhood tuberculous meningitis and miliary tuberculosis worldwide: a meta-analysis and assessment of cost-effectiveness. Lancet 367:1173-1180

19. van Leth F, van der Werf MJ, Borgdorff MW (2008) Prevalence of tuberculous infection and incidence of tuberculosis: a re-assessment of the Styblo rule. Bull World Health Organ $86: 20-26$

20. Tiemersma EW, van der Werf MJ, Borgdorff MW, Williams BG, Nagelkerke NJD (2011) Natural history of tuberculosis: duration and fatality of untreated pulmonary tuberculosis in HIV negative patients: a systematic review. PLoS One 6:e17601

21. Thompson BC (1943) Survival rates in pulmonary tuberculosis. Br Med J 2:721-721

22. Jindani A, Aber VR, Edwards EA, Mitchison DA (1980) The early bactericidal activity of drugs in patients with pulmonary tuberculosis. Am Rev Respir Dis 121:939-949

23. Loudon RG, Spohn SK (1969) Cough frequency and infectivity in patients with pulmonary tuberculosis. Am Rev Respir Dis 99:109-111

24. Davies C. The eradication of tuberculosis in Rhodesia: with particular reference to the Midlands and South Eastern Provinces. 1966.

25. Marx FM, Dunbar R, Enarson DA et al (2014) The temporal dynamics of relapse and reinfection tuberculosis after successful treatment: a retrospective cohort study. Clin Infect Dis 58:1676-1683

26. Marx FM, Floyd S, Ayles H, Godfrey-Faussett P, Beyers N, Cohen T (2016) High burden of prevalent tuberculosis among previously treated people in Southern Africa suggests potential for targeted control interventions. Eur Respir J 48:1227-1230

27. Dodd PJ, Gardiner E, Coghlan R, Seddon JA (2014) Burden of childhood tuberculosis in 22 high-burden countries: a mathematical modelling study. Lancet Glob Health 2:e453-e459

28. Dodd PJ, Sismanidis C, Seddon JA (2016) Global burden of drug-resistant tuberculosis in children: a mathematical modelling study. Lancet Infect Dis 16:1193-1201 
29. Horton KC, MacPherson P, Houben RMGJ, White RG, Corbett EL (2016) Sex differences in tuberculosis burden and notifications in low- and middle-income countries: a systematic review and meta-analysis. PLoS Med 13:e1002119

30. Stevenson CR, Forouhi NG, Roglic G et al (2007) Diabetes and tuberculosis: the impact of the diabetes epidemic on tuberculosis incidence. BMC Public Health 7:234

31. Corbett EL, Churchyard GJ, Clayton TC et al (2000) HIV infection and silicosis: the impact of two potent risk factors on the incidence of mycobacterial disease in South African miners. AIDS 14:2759-2768

32. Lin H-H, Ezzati M, Murray M (2007) Tobacco smoke, indoor air pollution and tuberculosis: a systematic review and meta-analysis. PLoS Med 4:e20

33. Lönnroth K, Williams BG, Cegielski P, Dye C (2010) A consistent log-linear relationship between tuberculosis incidence and body mass index. Int J Epidemiol 39:149-155

34. Lönnroth K, Jaramillo E, Williams BG, Dye C, Raviglione M (2009) Drivers of tuberculosis epidemics: the role of risk factors and social determinants. Soc Sci Med 68:2240-2246

35. Williams BG, Dye C (2003) Antiretroviral drugs for tuberculosis control in the era of HIV/ AIDS. Science 301:1535-1537

36. Ellis PK, Martin WJ, Dodd PJ. CD4 count and tuberculosis risk in HIV-positive adults not on ART: a systematic review and meta-analysis. PeerJ 2017; 5. https://doi.org/10.7717/ peerj. 4165

37. Williams BG, Gouws E, Somse P et al (2015) Epidemiological trends for HIV in Southern Africa: implications for reaching the elimination targets. Curr HIV/AIDS Rep 12:196-206

38. Williams BG, Korenromp EL, Gouws E, Schmid GP, Auvert B, Dye C (2006) HIV infection, antiretroviral therapy, and CD4+ cell count distributions in African populations. J Infect Dis 194:1450-1458

39. Wolbers M, Babiker A, Sabin C et al (2010) Pretreatment CD4 Cell slope and progression to AIDS or death in HIV-infected patients initiating antiretroviral therapy-The CASCADE Collaboration: a collaboration of 23 cohort studies. PLoS Med 7:e1000239

40. Touloumi G, Pantazis N, Pillay D et al (2013) Impact of HIV-1 subtype on CD4 count at HIV seroconversion, rate of decline, and viral load set point in European seroconverter cohorts. Clin Infect Dis 56:888-897

41. Dodd PJ, Prendergast AJ, Beecroft C, Kampmann B, Seddon JA (2017) The impact of HIV and antiretroviral therapy on TB risk in children: a systematic review and meta-analysis. Thorax 72:559-575

42. Dodd PJ, White RG, Corbett EL (2011) Periodic active case finding for TB: when to look? PLoS One 6:e29130

43. Toossi Z, Mayanja-Kizza H, Hirsch CS et al (2001) Impact of tuberculosis (TB) on HIV-1 activity in dually infected patients. Clin Exp Immunol 123:233-238

44. Samji H, Cescon A, Hogg RS et al (2013) Closing the gap: increases in life expectancy among treated HIV-positive individuals in the United States and Canada. PLoS One 8:e81355

45. Tanser F, Bärnighausen T, Grapsa E, Zaidi J, Newell M-L (2013) High coverage of ART associated with decline in risk of HIV acquisition in rural KwaZulu-Natal, South Africa. Science 339:966-971

46. Cohen MS, Chen YQ, McCauley M et al (2016) Antiretroviral therapy for the prevention of HIV-1 transmission. N Engl J Med 375:830-839

47. Guideline on when to start antiretroviral therapy and on pre-exposure prophylaxis for HIV. Geneva: World Health Organization; 2015.

48. Suthar AB, Lawn SD, del Amo J et al (2012) Antiretroviral therapy for prevention of tuberculosis in adults with HIV: a systematic review and meta-analysis. PLoS Med 9:e1001270

49. Lawn SD, Myer L, Edwards D, Bekker L-G, Wood R (2009) Short-term and long-term risk of tuberculosis associated with CD4 cell recovery during antiretroviral therapy in South Africa. AIDS 23:1717-1725

50. Nicholas S, Sabapathy K, Ferreyra C, Varaine F, Pujades-Rodríguez M, AIDS Working Group of Médecins Sans Frontières (2011) Incidence of tuberculosis in HIV-infected patients before and after starting combined antiretroviral therapy in 8 sub-Saharan African HIV programs. J Acquir Immune Defic Syndr 57:311-318 
51. Gupta A, Wood R, Kaplan R, Bekker L-G, Lawn SD (2012) Tuberculosis incidence rates during 8 years of follow-up of an antiretroviral treatment cohort in South Africa: comparison with rates in the community. PLoS One 7:e34156

52. McIlleron H, Meintjes G, Burman WJ, Maartens G (2007) Complications of antiretroviral therapy in patients with tuberculosis: drug interactions, toxicity, and immune reconstitution inflammatory syndrome. J Infect Dis 196:S63-S75

53. Nglazi MD, Bekker L-G, Wood R, Kaplan R (2015) The impact of HIV status and antiretroviral treatment on TB treatment outcomes of new tuberculosis patients attending co-located TB and ART services in South Africa: a retrospective cohort study. BMC Infect Dis 15:536

54. Giri PA, Deshpande JD, Phalke DB (2013) Prevalence of pulmonary tuberculosis among HIV positive patients attending antiretroviral therapy clinic. N Am J Med Sci 5:367-370

55. Recommendation on 36 months isoniazid preventive therapy to adults and adolescents living with HIV in resource-constrained and high TB- and HIV-prevalence settings: 2015 Update. World Health Organization, Geneva, p 2015

56. Akolo C, Adetifa I, Shepperd S, Volmink J (2010) Treatment of latent tuberculosis infection in HIV infected persons. Cochrane Database Syst Rev:CD000171

57. Golub JE, Saraceni V, Cavalcante SC et al (2007) The impact of antiretroviral therapy and isoniazid preventive therapy on tuberculosis incidence in HIV-infected patients in Rio de Janeiro, Brazil. AIDS 21:1441-1448

58. Ayele HT, van Mourik MSM, Debray TPA, Bonten MJM (2015) Isoniazid prophylactic therapy for the prevention of tuberculosis in HIV infected adults: a systematic review and metaanalysis of randomized trials. PLoS One 10:e0142290

59. Samandari T, Agizew TB, Nyirenda $S$ et al (2011) 6-month versus 36-month isoniazid preventive treatment for tuberculosis in adults with HIV infection in Botswana: a randomised, double-blind, placebo-controlled trial. Lancet 377:1588-1598

60. Houben RMGJ, Sumner T, Grant AD, White RG (2014) Ability of preventive therapy to cure latent Mycobacterium tuberculosis infection in HIV-infected individuals in high-burden settings. Proc Natl Acad Sci U S A 111:5325-5330

61. Langley I, Lin H-H, Egwaga S et al (2014) Assessment of the patient, health system, and population effects of Xpert MTB/RIF and alternative diagnostics for tuberculosis in Tanzania: an integrated modelling approach. Lancet Glob Health 2:e581-e591

62. Dowdy DW, Andrews JR, Dodd PJ, Gilman RH. A user-friendly, open-source tool to project impact and cost of diagnostic tests for tuberculosis. elife 2014; 3. https://doi.org/10.7554/ elife. 02565

63. Basu S, Andrews JR, Poolman EM et al (2007) Prevention of nosocomial transmission of extensively drug-resistant tuberculosis in rural South African district hospitals: an epidemiological modelling study. Lancet 370:1500-1507

64. Taylor JG, Yates TA, Mthethwa M, Tanser F, Abubakar I, Altamirano H (2016) Measuring ventilation and modelling $\mathrm{M}$. tuberculosis transmission in indoor congregate settings, rural KwaZulu-Natal. Int J Tuberc Lung Dis 20:1155-1161

65. Noakes CJ, Beggs CB, Sleigh PA (2004) Modelling the Performance of Upper Room Ultraviolet Germicidal Irradiation Devices in Ventilated Rooms: Comparison of Analytical and CFD Methods. Indoor Built Environ 13:477-488

66. RMGJ H, Dowdy DW, Vassall A et al (2014) How can mathematical models advance tuberculosis control in high HIV prevalence settings? Int J Tuberc Lung Dis 18:509-514

67. Bermejo A, Veeken H, Berra A (1992) Tuberculosis incidence in developing countries with high prevalence of HIV infection. AIDS 6:1203-1206

68. Schulzer M (1992) An estimate of the future size of the tuberculosis problem in sub-Saharan Africa resulting from HIV infection. Tuber Lung Dis 73:52-58

69. Dolin PJ, Raviglione MC, Kochi A (1994) Global tuberculosis incidence and mortality during 1990-2000. Bull World Health Organ 72:213-220

70. Schulzer M, Radhamani MP, Grzybowski S, Mak E, Fitzgerald JM (1994) A mathematical model for the prediction of the impact of HIV infection on tuberculosis. Int $\mathrm{J}$ Epidemiol 23:400-407 
71. Massad E, Burattini MN, Coutinho FAB, Yang HM, Raimundo SM (1993) Modeling the interaction between aids and tuberculosis. Math Comput Model 17:7-21

72. Naresh R, Sharma D, Tripathi A (2009) Modelling the effect of tuberculosis on the spread of HIV infection in a population with density-dependent birth and death rate. Math Comput Model 50:1154-1166

73. Roeger L-IW, Feng Z, Castillo-Chavez C (2009) Modeling TB and HIV co-infections. Math Biosci Eng 6:815-837

74. Kapitanov G (2015) A double age-structured model of the co-infection of tuberculosis and HIV. Math Biosci Eng 12:23-40

75. Basu S, Orenstein E, Galvani AP (2008) The theoretical influence of immunity between strain groups on the progression of drug-resistant tuberculosis epidemics. J Infect Dis 198:1502-1513

76. Basu S, Galvani AP (2009) The evolution of tuberculosis virulence. Bull Math Biol 71:1073-1088

77. Sergeev R, Colijn C, Murray M, Cohen T (2012) Modeling the dynamic relationship between HIV and the risk of drug-resistant tuberculosis. Sci Transl Med 4:135ra67

78. Porco TC, Small PM, Blower SM (2001) Amplification dynamics: predicting the effect of HIV on tuberculosis outbreaks. J Acquir Immune Defic Syndr 28:437-444

79. Murray M (2002) Determinants of cluster distribution in the molecular epidemiology of tuberculosis. Proc Natl Acad Sci U S A 99:1538-1543

80. Pretorius C, Dodd P, Wood R (2011) An investigation into the statistical properties of TB episodes in a South African community with high HIV prevalence. J Theor Biol 270:154-163

81. Escombe AR, DAJ M, Gilman RH et al (2008) The infectiousness of tuberculosis patients coinfected with HIV. PLoS Med 5:e188

82. Wood R, Johnstone-Robertson S, Uys P et al (2010) Tuberculosis transmission to young children in a South African community: modeling household and community infection risks. Clin Infect Dis 51:401-408

83. Uys P, Marais BJ, Johnstone-Robertson S, Hargrove J, Wood R (2011) Transmission elasticity in communities hyperendemic for tuberculosis. Clin Infect Dis 52:1399-1404

84. Andrews JR, Morrow C, Walensky RP, Wood R (2014) Integrating social contact and environmental data in evaluating tuberculosis transmission in a South African township. J Infect Dis 210:597-603

85. Andrews JR, Morrow C, Wood R (2013) Modeling the role of public transportation in sustaining tuberculosis transmission in South Africa. Am J Epidemiol 177:556-561

86. Dodd PJ, Looker C, Plumb ID et al (2016) Age- and sex-specific social contact patterns and incidence of Mycobacterium tuberculosis infection. Am J Epidemiol 183:156-166

87. Hughes G, Currie C, Corbett E. Modeling tuberculosis in areas of High HIV prevalence. In: Proceedings of the 2006 Winter Simulation Conference. 2006. https://doi.org/10.1109/ wsc. 2006.323116

88. Sánchez MS, Lloyd-Smith JO, Williams BG et al (2009) Incongruent HIV and tuberculosis co-dynamics in Kenya: interacting epidemics monitor each other. Epidemics 1:14-20

89. Blaser N, Zahnd C, Hermans S et al (2016) Tuberculosis in Cape Town: An age-structured transmission model. Epidemics 14:54-61

90. Pretorius C, Glaziou P, Dodd PJ, White R, Houben R (2014) Using the TIME model in Spectrum to estimate tuberculosis-HIV incidence and mortality. AIDS (28 Suppl 4):S477-S487

91. Agusto FB, Adekunle AI (2014) Optimal control of a two-strain tuberculosis-HIV/AIDS coinfection model. Biosystems 119:20-44

92. Kaur N, Ghosh M, Bhatia SS (2014) The role of screening and treatment in the transmission dynamics of HIV/AIDS and tuberculosis co-infection: a mathematical study. J Biol Phys 40:139-166

93. Sharomi O, Podder CN, Gumel AB, Song B (2008) Mathematical analysis of the transmission dynamics of HIV/TB coinfection in the presence of treatment. Math Biosci Eng 5:145-174 
94. Bacaër N, Ouifki R, Pretorius C, Wood R, Williams B (2008) Modeling the joint epidemics of TB and HIV in a South African township. J Math Biol 57:557-593

95. Wallengren K, Scano F, Nunn P, Margot B, Buthelezi S, Williams B, Pym A, Samuel EY, Mirzayev F, Nkhoma W, Mvusi L, Pillay Y. Resistance to TB drugs in KwaZulu-Natal: causes and prospects for control. 2011. https://arxiv.org/pdf/1107.1800.pdf.

96. Basu S, Friedland GH, Medlock J et al (2009) Averting epidemics of extensively drugresistant tuberculosis. Proc Natl Acad Sci U S A 106:7672-7677

97. Basu S, Stuckler D, McKee M (2011) Addressing institutional amplifiers in the dynamics and control of tuberculosis epidemics. Am J Trop Med Hyg 84:30-37

98. Heymann SJ (1993) Modelling the efficacy of prophylactic and curative therapies for preventing the spread of tuberculosis in Africa. Trans R Soc Trop Med Hyg 87:406-411

99. Guwatudde D, Debanne SM, Diaz M, King C, Whalen CC (2004) A re-examination of the potential impact of preventive therapy on the public health problem of tuberculosis in contemporary sub-Saharan Africa. Prev Med 39:1036-1046

100. Cohen T, Lipsitch M, Walensky RP, Murray M (2006) Beneficial and perverse effects of isoniazid preventive therapy for latent tuberculosis infection in HIV-tuberculosis coinfected populations. Proc Natl Acad Sci U S A 103:7042-7047

101. Basu S, Maru D, Poolman E, Galvani A (2009) Primary and secondary tuberculosis preventive treatment in HIV clinics: simulating alternative strategies. Int J Tuberc Lung Dis 13:652-658

102. Mills HL, Cohen T, Colijn C (2011) Modelling the performance of isoniazid preventive therapy for reducing tuberculosis in HIV endemic settings: the effects of network structure. J R Soc Interface 8:1510-1520

103. Kunkel A, Crawford FW, Shepherd J, Cohen T (2016) Benefits of continuous isoniazid preventive therapy may outweigh resistance risks in a declining tuberculosis/HIV coepidemic. AIDS 30:2715-2723

104. Baltussen R, Floyd K, Dye C (2005) Cost effectiveness analysis of strategies for tuberculosis control in developing countries. BMJ 331:1364

105. Laxminarayan R, Klein EY, Darley S, Adeyi O (2009) Global investments in TB control: economic benefits. Health Aff 28:w730-w742

106. Sánchez MS, Lloyd-Smith JO, Porco TC et al (2008) Impact of HIV on novel therapies for tuberculosis control. AIDS 22:963-972

107. Dowdy DW, Chaisson RE (2009) The persistence of tuberculosis in the age of DOTS: reassessing the effect of case detection. Bull World Health Organ 87:296-304

108. Mellor GR, Currie CSM, Corbett EL (2011) Incorporating household structure into a discreteevent simulation model of tuberculosis and HIV. ACM Trans Model Comput Simul 21:1-17

109. Yaesoubi R, Cohen T (2013) Identifying dynamic tuberculosis case-finding policies for HIV/ TB coepidemics. Proc Natl Acad Sci U S A 110:9457-9462

110. Azman AS, Golub JE, Dowdy DW. How much is tuberculosis screening worth? Estimating the value of active case finding for tuberculosis in South Africa, China, and India. BMC Med 2014; 12 https://doi.org/10.1186/s12916-014-0216-0

111. Dowdy DW, Chaisson RE, Moulton LH, Dorman SE (2006) The potential impact of enhanced diagnostic techniques for tuberculosis driven by HIV: a mathematical model. AIDS 20:751-762

112. Menzies NA, Cohen T, Lin H-H, Murray M, Salomon JA (2012) Population health impact and cost-effectiveness of tuberculosis diagnosis with Xpert MTB/RIF: a dynamic simulation and economic evaluation. PLoS Med 9:e1001347

113. Lin H-H, Langley I, Mwenda R et al (2011) A modelling framework to support the selection and implementation of new tuberculosis diagnostic tools. Int J Tuberc Lung Dis 15:996-1004

114. Langley I, Doulla B, Lin H-H, Millington K, Squire B (2012) Modelling the impacts of new diagnostic tools for tuberculosis in developing countries to enhance policy decisions. Health Care Manag Sci 15:239-253

115. Williams BG, Granich R, Chauhan LS, Dharmshaktu NS, Dye C (2005) The impact of HIV/ AIDS on the control of tuberculosis in India. Proc Natl Acad Sci U S A 102:9619-9624 
116. Granich RM, Gilks CF, Dye C, De Cock KM, Williams BG (2009) Universal voluntary HIV testing with immediate antiretroviral therapy as a strategy for elimination of HIV transmission: a mathematical model. Lancet 373:48-57

117. Bhunu CP, Garira W, Mukandavire Z (2009) Modeling HIV/AIDS and tuberculosis coinfection. Bull Math Biol 71:1745-1780

118. Dodd PJ, Knight GM, Lawn SD, Corbett EL, White RG (2013) Predicting the long-term impact of antiretroviral therapy scale-up on population incidence of tuberculosis. PLoS One 8:e75466

119. Pretorius C, Menzies NA, Chindelevitch L et al (2014) The potential effects of changing HIV treatment policy on tuberculosis outcomes in South Africa: results from three tuberculosisHIV transmission models. AIDS (28 Suppl 1):S25-S34

120. Currie CSM, Williams BG, Cheng RCH, Dye C (2003) Tuberculosis epidemics driven by HIV: is prevention better than cure? AIDS 17:2501-2508

121. Currie CSM, Floyd K, Williams BG, Dye C (2005) Cost, affordability and cost-effectiveness of strategies to control tuberculosis in countries with high HIV prevalence. BMC Public Health 5:130

122. Chindelevitch L, Menzies NA, Pretorius C, Stover J, Salomon JA, Cohen T. Evaluating the potential impact of enhancing HIV treatment and tuberculosis control programmes on the burden of tuberculosis. J R Soc Interface 2015; 12 https://doi.org/10.1098/rsif.2015.0146

123. Knight GM, Dodd PJ, Grant AD, Fielding KL, Churchyard GJ, White RG (2015) Tuberculosis prevention in South Africa. PLoS One 10:e0122514

124. Gilbert JA, Long EF, Brooks RP et al (2015) Integrating community-based interventions to reverse the convergent TB/HIV epidemics in rural South Africa. PLoS One 10:e126267

125. Gilbert JA, Shenoi SV, Moll AP, Friedland GH, Paltiel AD, Galvani AP (2016) CostEffectiveness of Community-Based TB/HIV Screening and Linkage to Care in Rural South Africa. PLoS One 11:e165614

126. Eaton JW, Menzies NA, Stover J et al (2014) Health benefits, costs, and cost-effectiveness of earlier eligibility for adult antiretroviral therapy and expanded treatment coverage: a combined analysis of 12 mathematical models. Lancet Glob Health 2:e23-e34

127. RMGJ H, Menzies NA, Sumner T et al (2016) Feasibility of achieving the 2025 WHO global tuberculosis targets in South Africa, China, and India: a combined analysis of 11 mathematical models. Lancet Glob Health 4:e806-e815

128. Trauer JM, Ragonnet R, Doan TN, McBryde ES (2017) Modular programming for tuberculosis control, the 'AuTuMN' platform. BMC Infect Dis 17:546

129. RMGJ H, Lalli M, Sumner T et al (2016) TIME Impact - a new user-friendly tuberculosis (TB) model to inform TB policy decisions. BMC Med 14:56

Open Access This chapter is licensed under the terms of the Creative Commons Attribution 4.0 International License (http://creativecommons.org/licenses/by/4.0/), which permits use, sharing, adaptation, distribution and reproduction in any medium or format, as long as you give appropriate credit to the original author(s) and the source, provide a link to the Creative Commons licence and indicate if changes were made.

The images or other third party material in this chapter are included in the chapter's Creative Commons licence, unless indicated otherwise in a credit line to the material. If material is not included in the chapter's Creative Commons licence and your intended use is not permitted by statutory regulation or exceeds the permitted use, you will need to obtain permission directly from the copyright holder. 